\title{
Monitoring of heavy metal levels in roadside dusts of Thessaloniki, Greece in relation to motor vehicle traffic density and flow.
}

\author{
Caroline Ewen ${ }^{\mathrm{a}}$, Maria A. Anagnostopoulou $^{\mathrm{b}}$, Neil I. Ward ${ }^{\mathrm{a}}$ \\ ${ }^{a}$ Department of Chemical Sciences, Faculty of Science, University of Surrey, \\ Guildford GU2 7XH, UK. \\ ${ }^{\mathrm{b}}$ Department of Geology, Faculty of Science, Aristotle University of Thessaloniki, \\ Thessaloniki 54124, Greece. \\ *Corresponding author's address: 7, Megalou Alexandrou, Nea Paralia, P.C. 54640, \\ Thessaloniki, Greece. Tel and Fax: 003-2310-810481. E-mail address: \\ managnos@auth.gr
}

\begin{abstract}
In recent years, the level of heavy metal pollution in urban areas has been of considerable concern. The principal source has been attributed to the motor vehicle and increasing inner city congestion, which has lead to a change and enlargement of transport stop-start zones. These areas of high traffic density are associated with an increased release of heavy metals into the adjacent residential or commercial areas. Seventy-five roadside dust samples were collected throughout the inner city and bypass motorway areas of Thessaloniki, Northern Greece. Samples were taken from arterial, major and minor roads, as well as the ring road, to compare and contrast the levels of heavy metals, namely $\mathrm{Cu}, \mathrm{Zn}, \mathrm{Cd}, \mathrm{Mn}$ and $\mathrm{Pb}$. Flame Atomic Absorption Spectroscopy (FAAS) was developed to quantitatively determine concentrations of both total element and geochemical fractionation, within the two dust particulate fraction sizes $<75 \mu \mathrm{m}$ and $75-125 \mu \mathrm{m}$. Acid digestion using Aqua Regia (3:1 conc. $\mathrm{HCl}: \mathrm{HNO}_{3}$ ) was employed for the total elemental analysis, a method that was validated through the use of certified reference compounds. Fractionation studies involved a three-step sequential extraction method performed on five selected samples (representatives of high, mid and low total elemental concentrations). The resultant solutions were analysed for lead and zinc levels to ascertain fractionation throughout the different geochemical fractions, thus assessing bioavailability. It was found that congestion/stop-start traffic patterns did influence and have led to increased levels of heavy metal deposition along inner city roads compared to levels observed on the new relief ring road.
\end{abstract}

\section{Keywords}

Heavy metals, pollution, FAAS, total elemental analysis, speciation analysis. 


\section{Introduction}

In today's modern society, urban developments and an ever-increasing traffic density have led to escalating amounts of motor vehicle pollution. During the past 25 years, the problem of heavy metal pollution was thought that lied within the inner city areas as well as motorways. In the first few minutes of a motor vehicle engine being engaged, the largest source of catalytic converter particulates is being deposited onto the road surface. As a result, several heavy metals are released, especially Pt, Pd and Rh (used as the catalysts).(Ward et al. 2004). Therefore, one of the major reasons behind the high heavy metal levels within inner cities is the 'stop-start' pattern that is often observed. Changes in traffic flow patterns and congestion in specific inner city areas (roundabouts, traffic lights/junctions) result in the possible release of many heavy metals, both from combustion particles and the wear-and-tear of the vehicle (especially tyres, brake linings etc.) (Charlesworth et al. 2003). Moreover, another important feature of inner city problems is the 'canyon' effect of buildings, which has a direct influence on dilution and dispersion pathways of the heavy metals released from motor vehicles (Namdeo et al. 1999). The effect of prevailing winds is not always evident within the environment of tall buildings and, as a result, particles tend to fall-out within the urban roadway 'canyon' resulting in higher heavy metal levels than found alongside motorways, where higher traffic densities are observed.

With regards to the urban/roadside environment the major contributor of pollution is the motor vehicle. It contains a complete spectrum of heavy and transition metals. In addition to the widely recognised exhaust emissions, there is also the wear of tyres, engine, chrome plating, brakes, weldings and bodywork (Rayson 1990), not to mention vehicle related contributors such as de-icers, accidental road spills and motorway surfaces (Hares and Ward 2004). All of the motorway-related elements 
such as $\mathrm{Cd}, \mathrm{Cr}, \mathrm{Cu}, \mathrm{Fe}, \mathrm{Mn}, \mathrm{Mo}, \mathrm{Ni}, \mathrm{Pb}, \mathrm{V}, \mathrm{Zn}, \mathrm{Al}, \mathrm{Pt}, \mathrm{Pd}$ and $\mathrm{Rh}$ are dangerous in themselves, but as with any type of pollution, it is the interaction with other compounds and media in the environment that can lead to the most harmful consequences. In 1990, the internal combustion engine accounted for $60 \%$ of total anthropogenic lead in environment (Rayson 1990). Today, eighteen years later, leaded petrol is no longer sold yet lead is still seen in roadside media. This can be often be directly related back to the availability of the metal.

Although many improvements have been made to reduce the amount of pollution from motor vehicles (unleaded petrol, catalytic converters, corrosion prevention etc.), these modifications combined with the increased volume and congestion on the roads counteract the benefits by simply providing new sources of different heavy metals (as illustrated by Pt from catalytic converters) (Ward et al. 2004; Rayson 1990).

In this study, road side dust was used as the 'pollutant monitor' due to its availability on all urban routes and its association with particulate matter released from motor vehicles. Lead, copper, cadmium, manganese and zinc were chosen for quantitative determination since these heavy metals are extremely toxic for the human organism. Lead is an element that can affect almost every organ and system in the body. The central nervous and skeletal systems, particularly in children, are especially vulnerable. Moreover, damage can also occur in the kidneys, the reproductive system and the brain. In a dust form, lead can be easily inhaled or swallowed by children, both of which have similar effects. Copper is not as dangerous as other heavy metals due to it being used extensively within the body but, like any other element, an excess is toxic. Copper will deposit in the brain and liver and lead to both physical and psychological problems. Hypoglycemia and anaemia are linked to copper excess, as are symptoms such as hyperactivity and learning difficulties in children. Cadmium is 
an especially toxic heavy metal that is listed as a suspected carcinogen. Inhaling high levels can damage the lungs and ingestion will irritate the stomach. Cadmium accumulates in the kidneys and is a possible cause of kidney disease. Other symptoms can include alopecia, anaemia, arthritis, osteoporosis, growth impairment and hypertension. Manganese is a metal that is most harmful when inhaled. By this route, it is transported directly to the brain without being metabolised, hence, an excess can lead to some neurological problems. Naturally, manganese is used in co-enzymes and, due to its metabolism being similar to that of iron, high levels can interfere with the absorption of dietary iron which leads to anaemia. Copper activity can also be affected through excess manganese. Zinc is an essential element that is used for enzymatic purposes. However, excess levels will interfere with the metabolism of iron and copper within the body. Large doses will lower manganese levels and increase susceptibility to autoimmune reactions. Further symptoms can include anaemia, growth retardation and increased low density lipoprotein (LDL) leading to raised cholesterol levels ( Dickerson and Horrath 1994). Listed above are some of the excess related toxicities of the metals examined in this study. It should be noted, though, other heavy metals will show similar symptoms and diseases, especially in terms of deficiencies in other essential metals within the body.

\section{Methodology}

\subsection{Sample Collection}

The focus of the present study was the city of Thessaloniki, Greece's second biggest city. This metropolitan area was chosen for the urban traffic pollution investigation, due to its having both major and minor transportation routes within the city and a new ring road built to by-pass the city. For analysis, 75 roadside dust samples were collected from Thessaloniki during a week in November 2006 under dry 
meteorological conditions (see Figure 1). Each sample was taken as a scoop sample and roughly filled a $10 \mathrm{ml}$ sample tube with screw lid. The roadside dust samples of Thessaloniki were categorised into four different road types: motorway/ringroad, arterial, major and minor roads.

The collected samples consisted of a variety of roadside dust particle sizes ranging from large grit to aerosol particles.

\subsection{Sample Preparation}

All roadside dust samples were initially dried and ground, using a pestle and mortar, to ensure homogenity. These were then sieved through $125 \mu \mathrm{m}$ and $75 \mu \mathrm{m}$ stainless steel sieves to create fractions $<75 \mu \mathrm{m}, 75-125 \mu \mathrm{m}$ and $>125 \mu \mathrm{m}$.

\subsection{Total Elemental Analysis}

It was decided to concentrate on the two smaller fractions, $<75 \mu \mathrm{m}$ and $75-125 \mu \mathrm{m}$, as the literature suggested these would yield the highest concentrations and they are known to be the most harmful if inhaled (Ward, 2004). Wet acid digestion by Aqua Regia (3:1, conc. $\left.\mathrm{HCl}: \mathrm{HNO}_{3}\right)$ was used for quantitative total elemental analysis. 0.2 $\pm 0.005 \mathrm{~g}$ of homogenised roadside dust sample was weighed out and transferred to a Kjeldahl tube. To this, $10 \mathrm{ml}$ of Aqua Regia was added before heating in a Kjeldahl heating mantle at $\sim 150^{\circ} \mathrm{C}$. Contents of the tube were transferred to a $50 \mathrm{ml}$ plastic Sterilin ${ }^{\mathrm{TM}}$ and with washing, the volume was made up to $25 \mathrm{ml}$ using DDW (Distilled, De-ionised Water). Samples were centrifuged at $3000 \mathrm{rpm}$ for 10 minutes and stored in the fridge at $4^{\circ} \mathrm{C}$ prior to analysis.

\subsection{Sequential Extraction}

When assessing the impact of a metal pollutant on the environment, it is often not adequate to just investigate total metal content. This derives from the fact that different geochemical forms of the same metal will interact and behave differently in 
relation to mobility and bioavailability (Stead et al. 2000). Sequential extraction is the method used to partition particulate metals depending on their affinity to certain soil types (Tessler et al. 1979). Although it is also described as 'speciation', the separation is due to geochemical forms as opposed to specific species, analysis of which would prove to be too difficult due to a amount of variables (Stead et al. 2000).

A range of different approaches have been developed and modified for this type of 'speciation'. The Rauret et al. 1999 extraction method involves fractionation relating to exchangeable, Fe-Mn oxide-bound, organic matter-bound and residual phases. Each step is more destructive than the previous. The exchangeable fraction involves the extraction of water soluble metals that are not specifically bound, hence the use of a mild reagent such as acetic acid. Fe-Mn oxides act as scavengers for trace metals so will bind to metals by adsorbing them onto their structure but are unstable in anoxic conditions, hence the use of a reducing agent such as hydroxylammonium chloride. Organic matter covers metals bound to anything organic including humic and fulvic acids. This matter is dissolved through oxidation by hydrogen peroxide and then released by ammonium acetate. The remaining content is the residual fraction (Tessler et al. 1979).

As with total analysis, roadside dust samples from both the $<75 \mu \mathrm{m}$ and $75-125 \mu \mathrm{m}$ size fractions were taken. The roadside dust samples chosen for additional analysis by sequential extraction were sample numbers 34, 35, 47, 50 and 68. Examination of the total elemental levels led to these samples being selected as representative of typical low $(47,50)$, middle $(68)$ and high $(34,35)$ heavy metal pollution containing samples. The extraction procedure followed is stated below:

(i) $1.00 \pm 0.01 \mathrm{~g}$ of homogenised roadside dust was placed in a $50 \mathrm{ml}$ Sterilin $^{\mathrm{TM}}$. To this, $40 \mathrm{ml}$ of $0.11 \mathrm{M}$ acetic acid was added and the mixture was shaken at $120 \mathrm{rpm}$ for 
4.5 hours. Following this, the Sterilin ${ }^{\mathrm{TM}}$ was centrifuged at $3000 \mathrm{rpm}$ for 5 minutes before the supernatant was removed using a Pasteur pipette (solution 1). The remaining roadside dust was washed twice by adding $20 \mathrm{ml}$ of DDW, shaking for 15 minutes and then centrifuging at $3000 \mathrm{rpm}$ for 5 minutes. Each time the supernatant was discarded;

(ii) To the remaining roadside dust, $40 \mathrm{ml}$ of $0.5 \mathrm{M}$ hydroxylammonium chloride was added and step (i) was repeated (solution 2). Furthermore, to the remaining roadside dust, $10 \mathrm{ml}$ of $30 \%$ hydrogen peroxide was added. The Sterilin ${ }^{\mathrm{TM}}$ was covered and left to digest at room temperature for 1 hour. The sample was then transferred and heated at $85^{\circ} \mathrm{C}$ for 1 hour in a PTFE squat beaker, to reduce the volume to $3 \mathrm{ml}$. A further 10 $\mathrm{ml}$ of $30 \%$ hydrogen peroxide was added, the Sterilin ${ }^{\mathrm{TM}}$ was covered and left to digest for 1 hour. At this time the cover was removed and digestion continued until the volume was approximately $1 \mathrm{ml}$. Finally, $50 \mathrm{ml}$ of ammonium acetate was added and the mixture was shaken at $120 \mathrm{rpm}$ for 4.5 hours before centrifuging as in steps 1 and 2 (solution 3).

2.5 Method of Analysis - Atomic Absorption Spectroscopy (AAS)

The AAS instrument used for analysis was a PerkinElmer AAnalyst400 (PerkinElmer Instruments LLC, Shelton, CT, USA). For all elements investigated, an air/acetylene flame was used. General Atomic Absorption Spectroscopy conditions for all elements were: acetylene flow rate $=2.50 \mathrm{l} / \mathrm{min}$; air flow rate $=10.00 \mathrm{l} / \mathrm{min}$; nebulizer flow rate $=1 \mathrm{ml} / \mathrm{min}$; burner head length $=80 \mathrm{~mm}$; dwell time $=2.0 \mathrm{~s}$ and repeat readings $=2$. Each element had a five point calibration run before sample analysis. The standards used, for each element, were AR blank solutions and were chosen based on the linear range recommended for the AAnalyst400 instrument. The calibration curves were set for linear through zero and correlations averaged at 0.99846 . 


\subsection{Method of Validation}

Three suitable Certified Reference Materials (CRM) were used to validate and compare results gained. The certified reference values for the reference materials used are: Ref1) NIST SRM 2557 Used Auto Catalyst (Monolith) $=13931 \mathrm{mg} / \mathrm{kg} \mathrm{Pb}$ and $1131 \mathrm{mg} / \mathrm{Kg}$ Pt, Ref2) NIST SRM 2556 Used Auto Catalyst (Pellets) $=6228 \mathrm{mg} / \mathrm{kg}$ $\mathrm{Pb}$ and $697.4 \mathrm{mg} / \mathrm{Kg}$ Pt and Ref3) Canmet Sudbury Matte PTM-1a $=7.31 \mathrm{mg} / \mathrm{Kg} \mathrm{Pt}$. The certified reference materials were selected when it was thought that Platinum might also be investigated. For the actual measurement process, this was not possible due to the limitations posed by the detection limit for atomic absorption spectroscopy and the concentrations present in the samples. However, analysis was still performed on all three reference materials as it was considered the content might be useful for sequential analysis purposes. Comparison between the values calculated and certified reference values for the Certified Reference Materials showed some differences; reference 1 calculated as $15483 \mathrm{mg} / \mathrm{kg} \mathrm{Pb}$ and reference 2 as $6858 \mathrm{mg} / \mathrm{kg} \mathrm{Pb}$. These differences showed that the results were not adequately matrix-matched and a correction factor was needed. The certified reference materials 1 and 2 values were plotted, certified against calculated. The graph equation was used to correct the lead concentration results. Due to lack of certified values for the other elements, the correction for lead was taken as a semi-quantitative correction for copper, cadmium, zinc and manganese. The corrected values were used for the analysis and interpretation of the data.

\subsection{Statistical Analysis}

All data from the total analyses were collated and the concentrations present in the original samples calculated using the matrix-matched calibration. The first study area was determination of any significant differences between the two particle fraction 
sizes $(<75 \mu \mathrm{m}$ and $75-125 \mu \mathrm{m})$. This was carried out through assessment of the distributions followed by comparisons of the variance, F-test and the mean, Student ttest (see Results and Discussion).

Value for each sample was the mean of three determinations.

\section{Results and Discussion}

\subsection{Copper}

Copper showed a similar pattern with regards to the roadside dust particle fraction sizes (see figure 2). There were, however, a couple of samples with high copper concentrations, which tended to be associated with the larger fraction sizes (75-125 $\mu \mathrm{m})$. This is in accordance with the fact that copper is linked with the wear-and-tear of brake pads (Rayson 1990). These higher Cu concentrations were found in samples 3 and 8 , followed by samples 25 and 33. Sample sites 3 and 25 were junctions, 8 was a minor inner city road in a green area and 33 was a tourist attraction. The highest $\mathrm{Cu}$ concentrations were found on a minor road but generally arterial roads provided the higher $\mathrm{Cu}$ levels (Fig 2). The major and minor roads seem to resulted in about the same levels of copper whilst the ring road consisted only of a minor source. Interestingly, since the ring road is a new fast tract transport route it might be postulated that the low copper levels are a result of no or minimal copper release from the brake-linings, as the vehicles are not under going stop-start flow conditions. With there being very few exit junctions, there is also no significant wear-and-tear of vehicle brakes.

\subsection{Zinc}

Apart from a couple of samples, zinc showed a general trend to be at higher levels in the smaller road side dust particle fraction sizes. There were two samples $(8,14)$ which showed very high Zn concentrations. Sample 14 was at a junction just outside 
the university and 8 was in the inner city. As far as the road type is concerned, it was not very easy to see any significant differences in figure 3 . Therefore figure 4 , without samples 8 and 14, was produced to gain a better general comparison. Arterial roads tended to have the higher $\mathrm{Zn}$ levels followed by minor and major showing similar values. The ring road samples were seen at the low concentration end (refer to the explanation for copper).

\subsection{Cadmium}

Cadmium was the element that showed the clearest divide in road side dust particle fraction sizes in terms of the total elemental concentrations observed ( see figure 5). This implies that the exhaust emissions of cadmium are greater than that which would be expected from a tire wear contribution. In nearly all the samples (except two) the smaller particle size fraction was found to have the highest Cd levels. The two most highly contaminated samples, at sites 32 and 34, were situated on the arterial coastal road. However, apart from these two samples, there was not much spread in $\mathrm{Cd}$ concentrations throughout the study sites, especially for the smaller particle size fraction. The arterial and minor roads might be seen to have higher $\mathrm{Cd}$ levels but this was only a marginal observation. The lowest $\mathrm{Cd}$ concentrations were found in two suburban roads, samples 16 and 75, along with sample 60, which was located alongside the ring road. These findings are of great importance as cadmium is considered to be a very toxic heavy metal in terms of human health. The fact that the smaller road side dust particles $(<75 \mu \mathrm{m})$ have the higher levels of $\mathrm{Cd}$ contamination suggests that roadside dust could pose a serious health risk in urban environments. This is because the smaller particle sizes have the potential to be taken up by the lungs. The possible sources of $\mathrm{Cd}$ are limited as reflected in the narrow spread across the sampling sites. Although the wear-and-tear of tyres is recognised as a major 
source of cadmium alongside roadways, this study suggests that maybe the combustion of fuel (especially diesel) and oil/lubricants, which are known to contain trace levels of cadmium, may be a significant source of $\mathrm{Cd}$ as found in roadside dust within the Thessaloniki area.

\subsection{Manganese}

Manganese was another element that illustrated a tendency to be more highly concentrated in the smaller road side dust particle fractions. Figure 6 shows a consistency in the Mn levels of samples taken from the Thessaloniki area. Highest Mn concentrations were observed in sample 32 , the coastal roadside dust sample, and samples 49 and 50. These two samples were from the ring road, which goes against the trends of the rest of the elements.

\subsection{Lead}

It can be seen from figure 7 that samples varied very little in lead concentrations between the fraction sizes. Furthermore, the graph highlights the arterial roads as being the ones with the highest lead concentrations in both fractions, though closely followed by major roads with the second highest levels. Minor roads are next and then the ring road following very closely behind.

This suggests that traffic flow patterns have an influence on the road side dust lead concentrations, not just traffic density. The highest concentrations were found in road side dust sample 25, which was collected at a site being a junction of several different road types. These high lead concentrations were observed in both fractions. The lowest concentrations were seen in samples 48 and 53, both of which were located in the suburbs. A possible explanation for this trend is not obvious in that the major source of lead, being added lead to fuel, is no longer in use in Greece. Residual lead from this source would not be expected to be evident in the present roadside dust 
samples, due to dust being considered a short-term contamination indicator (Xiangdong et al. 2001). The observed trend does show that the highest lead levels were found in busy/high traffic density roadways, namely the arterial roads. It is possible that modern cars via the wear-and-tear of wheel bearings are still releasing lead, but no evidence has been reported in the literature indicating this as a significant anthropogenic source in the urban environment. The data of this study suggests that this might be possible in zones of vehicle acceleration and de-acceleration. However, if the release of lead were from wheel bearings it would be expected that the particle size profile would be higher in the smaller particle sizes $(<75 \mu \mathrm{m})$, but the Student $\mathrm{t}$ test confirmed no significant difference in the lead content of the two particle size fraction groups. It must therefore be concluded that whilst lead is high in these arterial and possible high traffic density areas, the source could be wheel bearings and other anthropogenic inputs (as yet not identified). The overall results for Thessaloniki showed a significant improvement in the levels of lead alongside roadways since the 1981 and 1998 studies (Anagnostopoulos 1981; Anagnostopoulou and Day 2006) which had exhibited mean lead values of $1150 \mathrm{mg} / \mathrm{Kg}$ and $519 \mu \mathrm{g} / \mathrm{g}$ respectively.

Table gives a summary of all the elemental means for the separate particle fractions and a mean of the two fractions. Also listed in the Table are comparisons with similar reported studies from other cities and the published mean elemental concentrations in their roadside dust. It should be noted that these studies were undertaken at different times (Istanbul 2004, Hong Kong 2001 and London 1991) which account for example, for the high levels of lead observed in the early London study before the removal of leaded petrol. The difference in elemental content emphasises that roadside dust is often an indicator of short-term contamination from vehicles (Xiangdong et al. 2001). 


\subsection{Speciation Analysis - Sequential Extraction or Fractionation}

Two sequential analyses were done for lead and zinc. Lead was selected because of the concerns about residual levels of lead following the removal of leaded petrol; lead was a pollutant that was of significant concern about a decade ago. Since leaded petrol has been phased out it has been observed that lead levels in the roadside dust are lower but the question arises about the mobility of lead in the various environmental media, like dust, soil, plants and the aquatic ecosystems. It has already been seen in the present study that the lead content of roadside dust samples in Thessaloniki is fairly evenly distributed through both particle size fractions. This raises the question, maybe there is a different source of lead (such as wheel bearings or oil combustion products) or even that the roadside dust samples have input from a local domestic or commercial source (paint release as houses are refurbished, local small metal working sites). Zinc was chosen because of an increasing concern about the use of zinc-based compounds as lubricant additives, the potential poisoning of catalytic converters and enhanced release of zinc into the environment where it may have a detrimental effect on aquatic ecosystems. Furthermore, zinc was shown to exhibit vastly different concentrations in a selection of roadside dust samples as shown in Figure 2. It was therefore thought that the distribution of zinc through the geochemical fractions might be interesting and lead to explanations as to whether there are differing chemical forms of zinc whose origins are from various sources of the car (metal work, paint, exhaust system, tyres, oil additives, etc). Incidentally, the two samples that were chosen to represent high elemental concentrations $(34,35)$ were both located on the coastal road. Samples reflecting low metal concentrations $(47,50)$ were both located on the ring road (a new construction with very few exit points or traffic congestion problems) and the mid metal concentration sample (68) 
was from a crossroad. These samples were selected by inspection of the overall elemental concentrations of the roadside dust samples ( see figures 2 to 7 ) rather than by the location. The sequential extraction or fractionation graphs ( see figures 8 and 9) were calculated using both total and sequential data. The three solutions, representing the exchangeable, Fe-Mn oxide and organic fractions were summed and then subtracted from the total elemental content to calculate the elemental levels in the residual fraction. All these sequential extraction fractions were then calculated to provide an estimate of the percentage of the total elemental level recorded relative to the total elemental analysis.

\subsubsection{Lead}

Lead showed quite a significant difference in the sequential fractionation pattern between the samples classed as having generally high lead concentrations $(34,35)$ and those classed as having mid (68) or low Pb concentrations $(47,50)$ (see figure 8). None of the samples, except sample $47(75-125 \mu \mathrm{m})$ showed any real association with the organic fraction. The roadside dust samples $(34,35)$ containing higher total lead levels showed a high affinity for the residual fraction. This might be expected as the roadside dust samples having higher lead levels may still reflect input from the use of leaded petrol. This also confirms that lead is not very mobile in soils and sediments (Stead et al 2000), and therefore in the present study a similar observation is noted for roadside dusts. The other samples showed quite a high percentage of lead found in the exchangeable fraction. As far as particle size fraction differences, there was a similar split between the high and mid/low samples. The roadside dust samples containing high lead levels showed that the larger particle size fractions contained lead that had a high affinity for the residual fraction whereas the mid/low samples showed this reversed (see figure 8). However, overall the roadside dust samples reflected a 
tendency to have lead in the residual fraction for the larger particle sizes. This might also be expected in that residual matter is more likely to be the larger particles that are not so easily dispersed. Furthermore, the three certified reference materials showed differing sequential extraction patterns from each other and the road side dust samples under investigation. Reference materials 1 and 2 (Used Auto Catalyst) contained lead in the exchangeable and $\mathrm{Fe} / \mathrm{Mn}$ oxide fractions, which is similar to the roadside dust samples from roadways that have low or mid lead levels (sites 47, 50 and 68). The Canmet Sudbury Matte PTM- 1a certified reference material 3 (which is physically different in that it is very fine black powdered, origin unknown) is entirely different with the lead content being associated mainly with the residual fraction. This is similar to the roadside dust samples from sites 34 and 35, which contained some of the highest roadside dust lead levels, irrespective of particle size. These trends tend to suggest that there is a possibility that lead found in the road side dust samples of Thessaloniki not only varies in magnitude as a result of release from vehicles using the different road types (as a function of traffic density and flow conditions), but may also reflect different sources, and chemical forms, of lead.

\subsubsection{Zinc}

Figure 9 shows that zinc in roadside dust samples also exhibited a very low affinity for the organic phase, although maybe more (percentage wise) than lead. In summary, all of the road side dust samples showed a similar sequential extraction pattern throughout, with most of the zinc found in the following order: exchangeable $>\mathrm{Fe}-$ Mn oxide $>$ residual $>$ organic. This suggests that zinc may be very mobile in the environment or has an affinity to be associated with clay particles (Fe-Mn oxide fraction). This observation has also been found with zinc in motorway storm water drainage sediments (Stead et al 2000). Interestingly, the extraction or fractionation 
pattern for zinc was similar to that for lead in the low and mid lead level road side dust samples. Similarly, as with lead, there was no observable difference in the zinc sequential extraction pattern between road side dust particle size fractions. However, there was a significant difference in the zinc pattern between the road side dust samples from Thessaloniki and the three certified reference materials. Once again, the Canmet Sudbury Matte PTM-1a certified reference material 3 was slightly different from the other two Used Auto Catalyst materials, having little or no percentage of zinc associated with the Fe-Mn oxide fraction. This material contained zinc primarily associated with the exchangeable and residual fractions. Clearly, the reference materials and roadside dust samples contained different chemical forms of zinc. Although the total zinc analysis of the roadside dust samples showed that a couple of sampling sites had higher zinc burdens (sites 8 and 14), the samples selected for the sequential extraction study (sites 34, 35, 47, 50, 68) did not appear to have any obvious variation in chemical forms of zinc. Therefore, this study can not provide any details about the possible sources that contribute to the release of zinc from motor vehicles (metal work, paint, exhaust system, tyres, oil additives, etc) (Rayson 1990; Hares and Ward 2004).

Synoptically, a wet acid digestion method was used, with the use of Aqua Regia and Kjeldahl tubes, for total elemental analysis. It was decided that the three step sequential extraction used by Rauret et al. 1999 would be used to help provide geochemical and bioavailability data. The calibration method used to produce the Atomic Absorption Spectroscopy results was seen to not be matrix-matched, leading to the need for a modified semi-quantitative matrix-matched calibration method using the Certified Reference Materials. It is postulated that this method not only corrects for the matrix contribution in the analysis, but also reflects the efficiency of the 
digestion method relative to the roadside dust samples. These corrected values were used for statistical and graphical interpretations of the results.

Data from the total elemental analyses were carried out first. According to calculated values using the F-test, copper was the only element that showed a statistically significant difference in the sample variance between the two fraction sizes, with an $\mathrm{F}_{\text {cal }}$ value of 2.098 which is significantly different at the probability, $\mathrm{P}<0.001$ or 99.9\% confidence limit ( $\mathrm{F}_{\text {crit }}$ of 2.07$)$. Zinc and cadmium showed a significant difference at the $\mathrm{P}<0.05$ or $95 \%$ confidence limit. Only lead and manganese showed no significant difference at the $\mathrm{P}<0.05$ or $95 \%$ confidence limit. Once the F-test results were known, a Student t-test were carried out on the same data to assess whether there was any significant difference in the mean values between the two fraction sizes. Cadmium and manganese showed a statistically significant difference at the $\mathrm{P}<0.001$ or $99.9 \%$ confidence level, between the mean values of the two fraction sizes. Lead, copper and zinc showed no significant difference, at the $\mathrm{P}>0.05$ or $95 \%$ confidence limit. Fraction size is generally related to the source of the metal; smaller being associated with exhaust emissions and the engine, whereas larger particles with general wear-and-tear sources from all over the car's body. The results suggest that cadmium and manganese are being deposited more heavily through emissions while zinc, lead and copper are being deposited through several sources.

The other subcategory that was used to compare samples was different road types. The sample sites were divided, according to the map of Thessaloniki (see figure 1), into ring road, arterial, major and minor roads. The minor roads were also noted to have an average 10-100 cars/hr as opposed to the other roads which had an average density of $>100 \mathrm{cars} / \mathrm{hr}$. This comparison enabled variations in traffic density and flow to be considered. In general, it was seen that the arterial roads had the highest 
concentrations followed by major, minor and then the ring road (Figures 2-7). Lead showed a greater affinity, typically, with the arterial and major roads as opposed to the minor roads and ring road ( see figure 7). Copper was highest on the arterial roads followed by the major and minor roads before the ring road (see figure 2). For cadmium and manganese, distributions were almost even throughout the different road types (see figures 5 and 6). This implies that $\mathrm{Cd}$ and $\mathrm{Mn}$ are influenced by traffic flow patterns as opposed to the traffic density. It should also be noted that many of the highest metal concentrations were located on junctions, also supporting the notion of stop-start driving conditions being a major factor in the relationship of traffic to pollution levels.

From the sequential extractions, lead showed a large difference in distribution among the selected samples (see figure 8). It is difficult to say whether this pattern for $\mathrm{Pb}$ is due to location or reflects the magnitude of the metal in the samples. Samples 34 and 35 (highly $\mathrm{Pb}$ concentrated samples) showed the more expected association with the residual phase. The fact that the other samples were much more highly associated with the exchangeable fraction is more worrying as this suggests that the lead is favourable for being bioavailable as it is in a mobile phase. Obviously factors such as the chemical form and environment (pH etc.) would also affect the bioavailability. Zinc displayed a much more uniform picture across the samples ( see figure 9). Most Zn was located in the exchangeable and Fe-Mn oxide fractions and, similar to lead, little was residing in the organic phase. As zinc concentrations got lower, there was a slightly stronger affinity for the residual and weaker to the exchangeable fractions.

\section{Conclusions}


1. The sample comparison enables variations in traffic density and flow to be considered. The arterial streets had the highest metal concentrations followed by major, minor and ring roads.

2. The highest metal concentrations were in junctions, supporting the notion of stopstart driving conditions being a major factor in the relationship of traffic to pollution levels.

3. Both Cadmium and Manganese showed higher total levels in the smaller fraction sizes $(<75 \mu \mathrm{m})$, implying that their major release source is exhaust emissions. Lead, copper and zinc were all exhibited higher levels in the inner city routes. This can be related back to the wear-and-tear of vehicle components as a result of the stop-start traffic patterns (brake pads etc).

4. Fractionation studies revealed the presence of different chemical forms and sources of lead. Moreover, fractionation of road side dust samples for zinc showed less variation, with exchangeable and Fe-Mn oxide fractions showing the highest levels.

5. Motorway ring road, which displays few acceleration/de-acceleration points, was seen to have lower levels of heavy metals. Both lead and Zinc have shown to be in chemical forms that are bioavailable to ecosystems.

6. Last but not least, lead concentrations exhibited a remarkable decrease since 1981 lead study of Thessaloniki (Anagnostopoulos 1981) obviously as a result of the exclusion of leaded petrol.

\section{Acknowledgements}

The authors wish to acknowledge the scientific contributions made by Sarah Hill, Claire Stone and Judith Peters with regards to the operation of the instruments. 


\section{References}

Anagnostopoulos, A. (1981). Lead in some streets in Thessalonica. In:

Anagnostopoulos, A. (Ed.), Proceedings of First International Conference of Environmental Pollution, 21 September-25 September, (pp.190-202) Thessaloniki, Greece.

Anagnostopoulou, M.A., Day P.J. (2006). Lead concentrations and isotope ratios in street dust in major cities in Greece in relation to the use of lead in petrol. Science of the Total Environment, 367, 791-799.

Charlesworth, S., Everett, M., McCarthy, R., Ordonez A., DeMiguel, E. ( 2003). A comparative study of heavy metal concentration and distribution in deposited street dusts in a large and a small urban area: Birmingham and Coventry, West Midlands, UK. Environment International, 29 (5), 563-573.

Dickerson, O.B., \& Horrath, E. (1994). Occupational medicine. Zenz Carl. Fergusson, E.J. (1990). The heavy elements : Chemistry, Environmental impact and health effects, Pergamon Press.

Hares, R.J., Ward, N.I.( 2004). Sediment accumulation in newly constructed vegetative treatment facilities along a new major road. Science of the Total Environment, 334-335, 473-479.

Namdeo, A.K., Colls, J.J., Baker, C.J. (1999). Dispersion and re-suspension of fine and coarse particulates in an urban street canyon. Science of the Total Environment, $235(1-3), 3-13$.

Rayson, M.J.T. (1990). An investigation into the effects of vehicular pollution on the U.K. and North American environments. MSc. Dissertation. Department of Chemical Sciences, University of Surrey, UK. 
Rauret, G., Lopez-Sanchez, J.F., Sahuquillo, A., Rubio, R., Davidson, C., Ure, A., Quevauviller, P. (1999). Improvement of the BCR three step sequential extraction procedure prior to the certification of the new sediment and soil reference materials. Journal of Environmental Monitoring, 1, 57-61.

Sezgin, N.H., Ozcan, K., Demir, G., Nemlioglu, S., Bayat, C. ( 2004). Determination of heavy metal concentrations in street dusts in Istanbul E-5 highway. Environment International $29(7)$.

Stead, K., Hares, R.J., Ward, N.I. ( 2000). Sequential extractions, fractionation studies-What are they defining? In: J. Nriagu (Ed.), Eleventh Annual International Conference on Heavy Metals in the Environment, University of Michigan, School of Public Health (pp.1-27). Ann Arbor.

Tessler, A., Campell, P.G.C., Bisson M. (1979). Sequential extraction procedure for the speciation of particulate trace metals. Analytical Chemistry 51 (7).

Thorton I. 1991. Metal contamination of soils in urban areas. In: P.Bullock and P.J. Gregory (Ed.), Soils in the urban environment ( pp. 47-75). Blackwell.

Ward, N.I., Dudding, A., Lyndon, M. (2004). Platinum emissions and levels in motorway dust samples: influence of traffic characteristics. Science of the Total Environment, 334-335, 457-463.

Xiangdong, L., Chi-sun, P., Pui Sum, L. ( 2001). Heavy metal contamination of urban soils and street dusts in Hong Kong. Applied Geochemistry, 16 (11-12), 1361-1368. 
Table Summary of the elemental mean values for the different roadside dust particle fractions in this study relative to values reported in the literature ( ${ }^{\mathrm{a}}$ Sezgin et al. 2004;

${ }^{b}$ Xiangdong et al. 2001; ${ }^{c}$ Thorton 1991).

\begin{tabular}{|c|c|c|c|c|c|c|c|c|c|c|}
\hline & \multicolumn{2}{|c|}{$\mathrm{Pb}(\mathrm{mg} / \mathrm{kg})$} & \multicolumn{2}{|c|}{$\mathrm{Cu}(\mathrm{mg} / \mathrm{kg})$} & \multicolumn{2}{|c|}{$\mathrm{Mn}(\mathrm{mg} / \mathrm{kg})$} & \multicolumn{2}{|c|}{$\mathrm{Zn}(\mathrm{mg} / \mathrm{kg})$} & \multicolumn{2}{|c|}{$\mathrm{Cd}(\mathrm{mg} / \mathrm{kg})$} \\
\hline Fraction $(\mu \mathrm{m})$ & $<75$ & $75-125$ & $<75$ & $75-125$ & $<75$ & $75-125$ & $<75$ & $75-125$ & $<75$ & $75-125$ \\
\hline Mean & 206.5 & 206.9 & 255.4 & 282.9 & 211.0 & 173.0 & 515.4 & 410.3 & 5.1 & 2.9 \\
\hline $\begin{array}{l}\text { Total Mean of } \\
\text { both fractions. }\end{array}$ & \multicolumn{2}{|c|}{206.7} & \multicolumn{2}{|c|}{269.2} & \multicolumn{2}{|c|}{192.0} & \multicolumn{2}{|c|}{462.9} & \multicolumn{2}{|c|}{4.0} \\
\hline Istanbul $^{\mathrm{a}}$ & \multicolumn{2}{|c|}{211.88} & \multicolumn{2}{|c|}{208.49} & \multicolumn{2}{|c|}{397.90} & \multicolumn{2}{|c|}{526.81} & \multicolumn{2}{|c|}{1.91} \\
\hline Hong Kong $^{\text {b }}$ & \multicolumn{2}{|c|}{181} & \multicolumn{2}{|c|}{173} & \multicolumn{2}{|c|}{ - } & \multicolumn{2}{|c|}{1450} & \multicolumn{2}{|c|}{3.77} \\
\hline London $^{c}$ & \multicolumn{2}{|c|}{1354} & \multicolumn{2}{|c|}{115} & \multicolumn{2}{|c|}{ - } & \multicolumn{2}{|c|}{513} & \multicolumn{2}{|c|}{4.2} \\
\hline
\end{tabular}


Fig. 1 Map of the city of Thessaloniki showing the sampling area into the black circle. (Types of road: ring road, arterial road, major road and minor road).

Fig. 2 Variation in copper content due to road dust particle fraction size and road type.

Fig. 3 Variation in zinc content due to road dust particle fraction size and road type.

Fig. 4 Variation in zinc content due to fraction size and road type (without samples 14 and 8).

Fig. 5 Variation in cadmium content due to road dust particle fraction size and road type.

Fig. 6 Variation in manganese content due to road dust particle fraction size and road type.

Fig. 7 Variation in lead content due to road dust particle fraction size and road type.

Fig. 8 Sequential fractionation of lead in selected roadside dust samples and certified reference materials (CRM) (with differing particle sizes, $<75 \mu \mathrm{m}$ and 75 to $125 \mu \mathrm{m}$ ).

Fig. 9 Sequential fractionation of zinc in selected roadside dust samples and certified reference materials (CRM) (with differing particle sizes, $<75 \mu \mathrm{m}$ and 75 to $125 \mu \mathrm{m}$ ). 


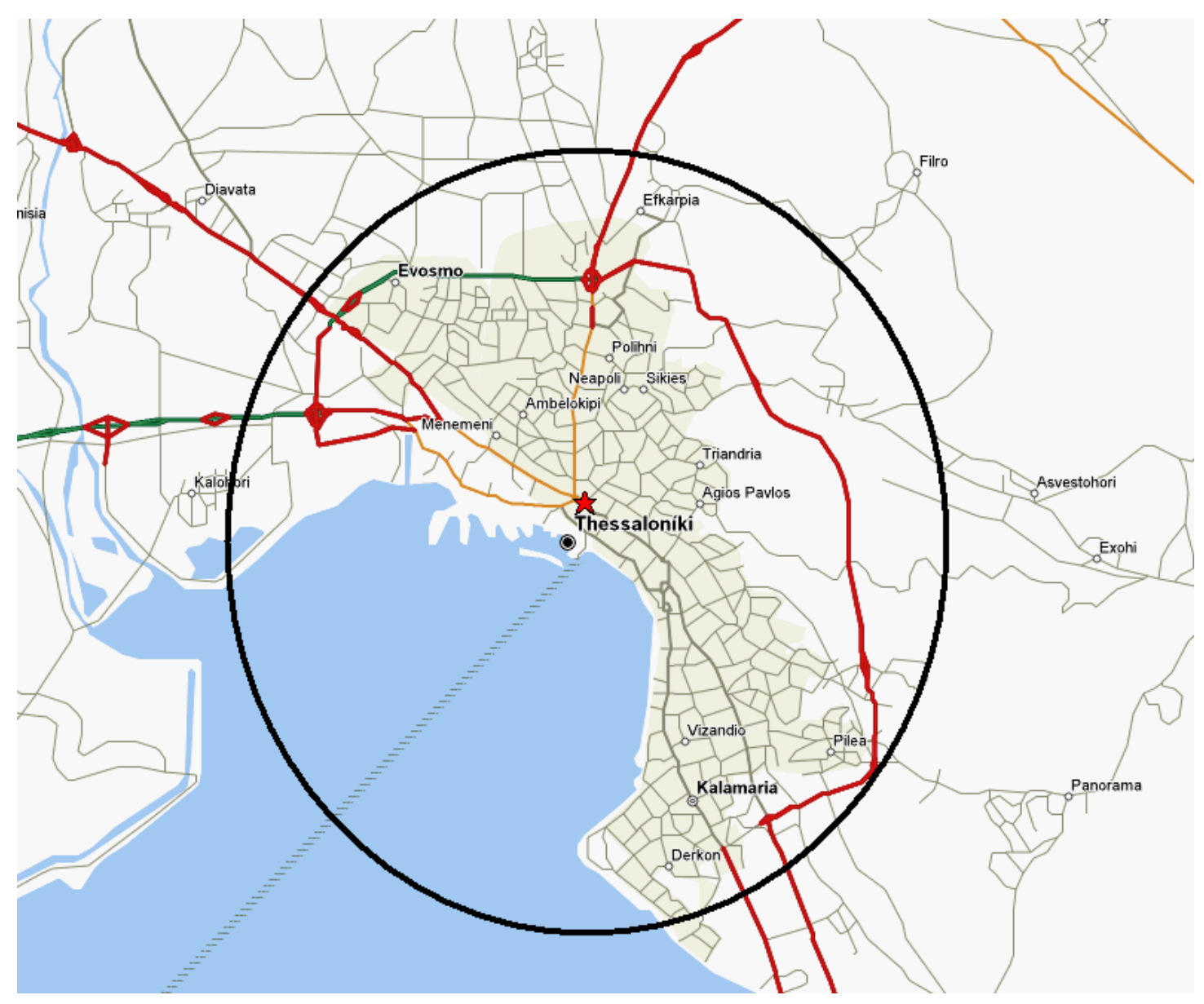

Fig. 1 

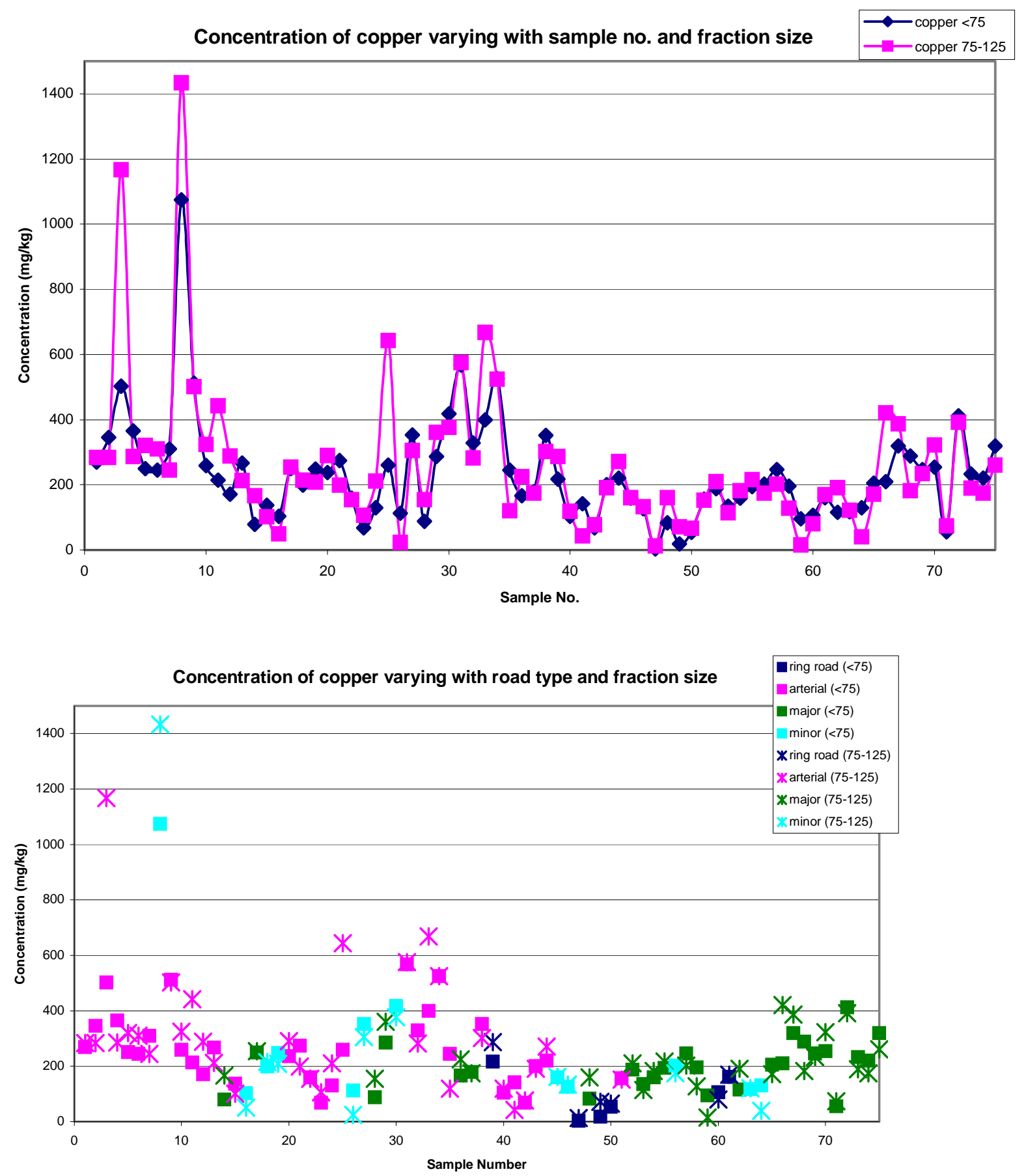

Fig. 2 

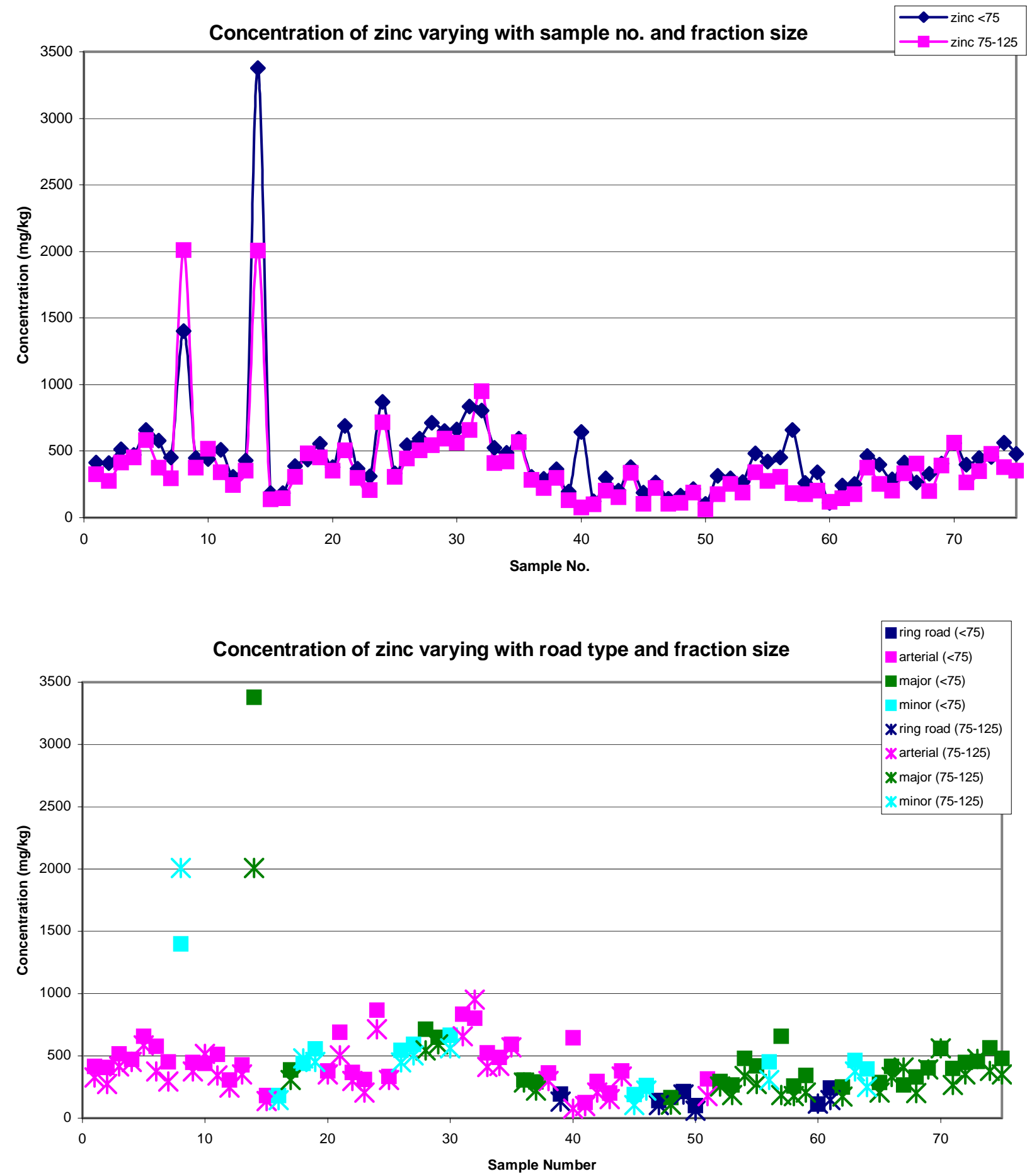

Fig. 3 


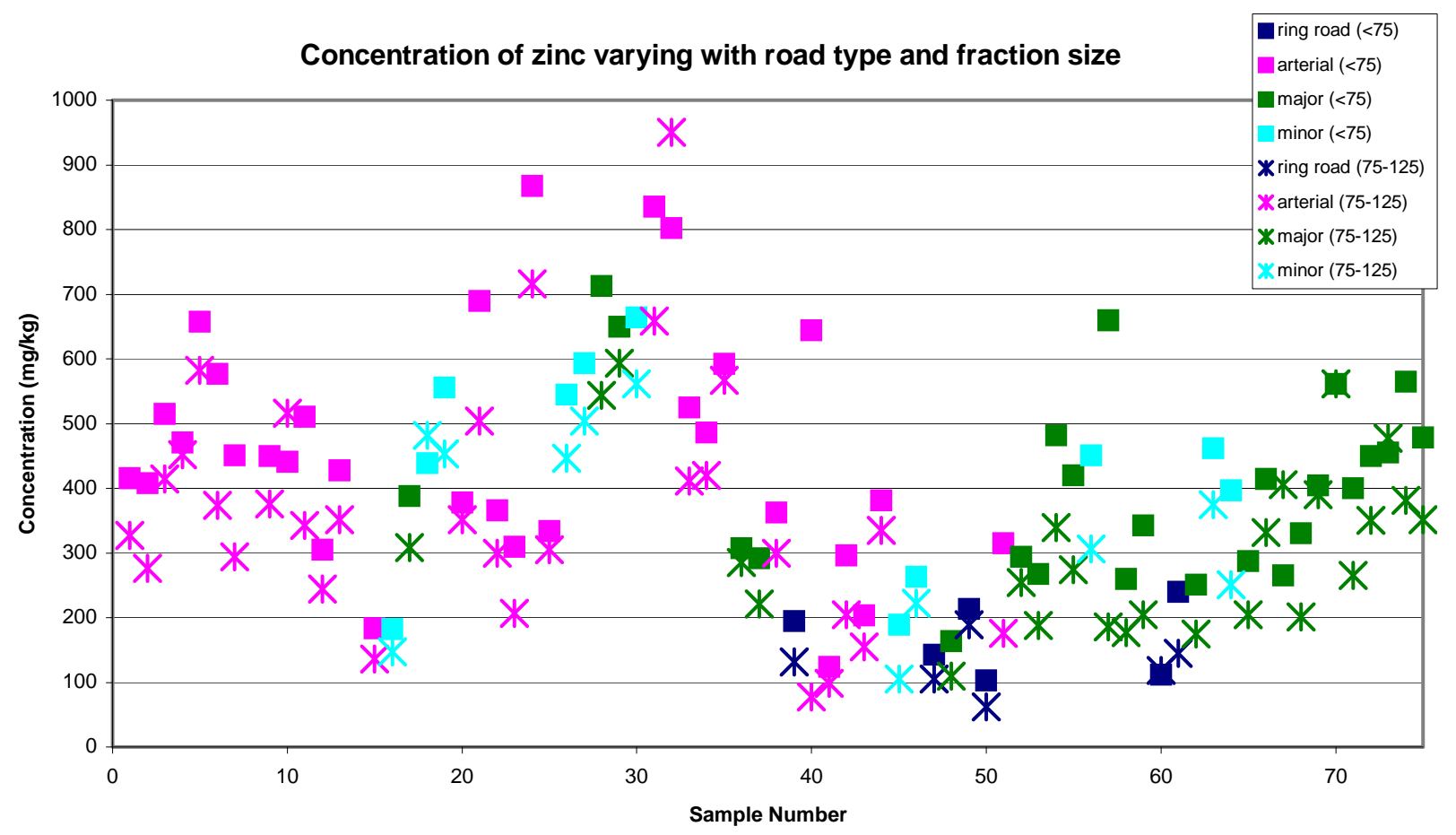

Fig. 4 

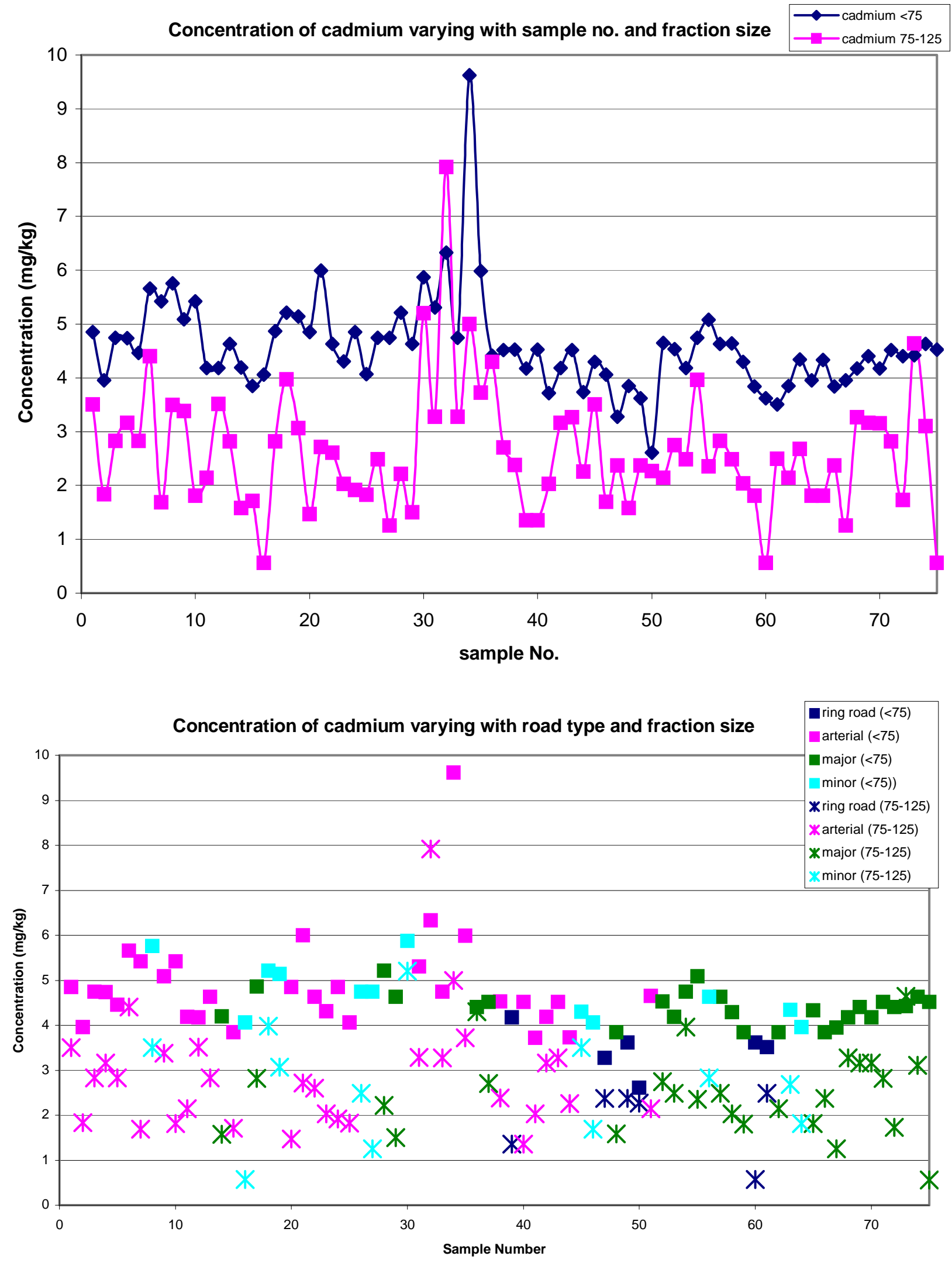

Fig. 5 

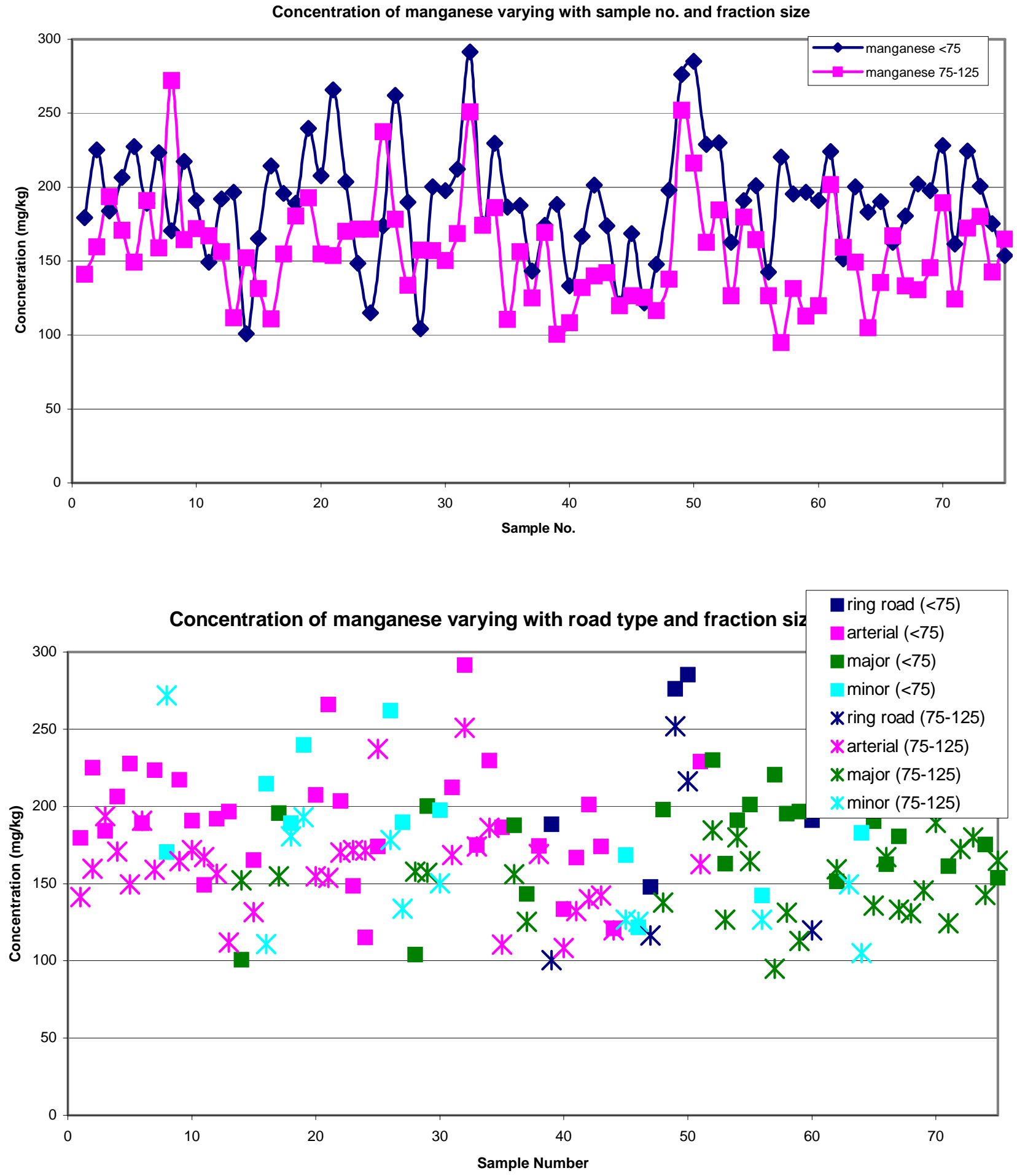

Fig. 6 

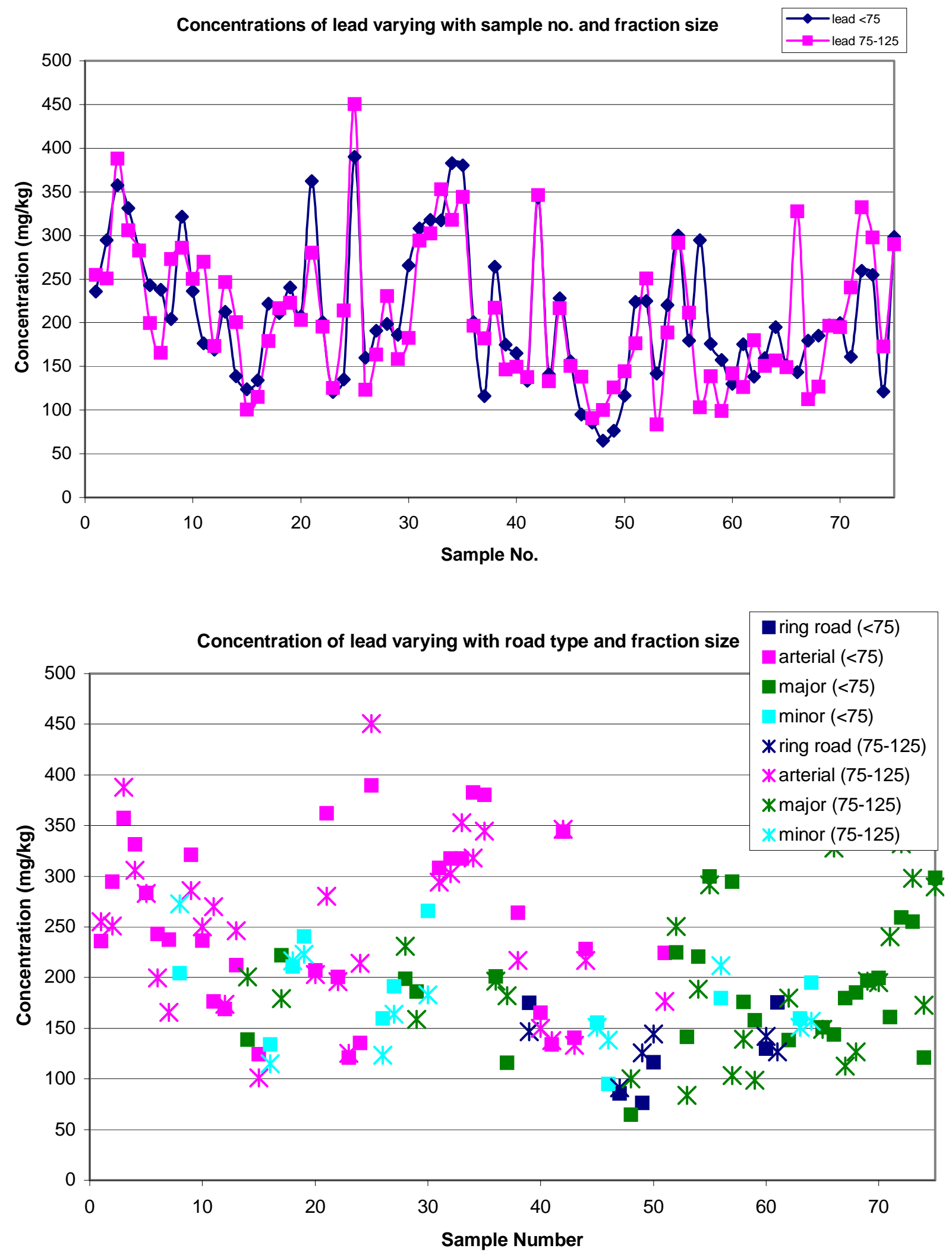

Fig. 7 
Distribution of $\mathrm{Pb}$ through the different fractions

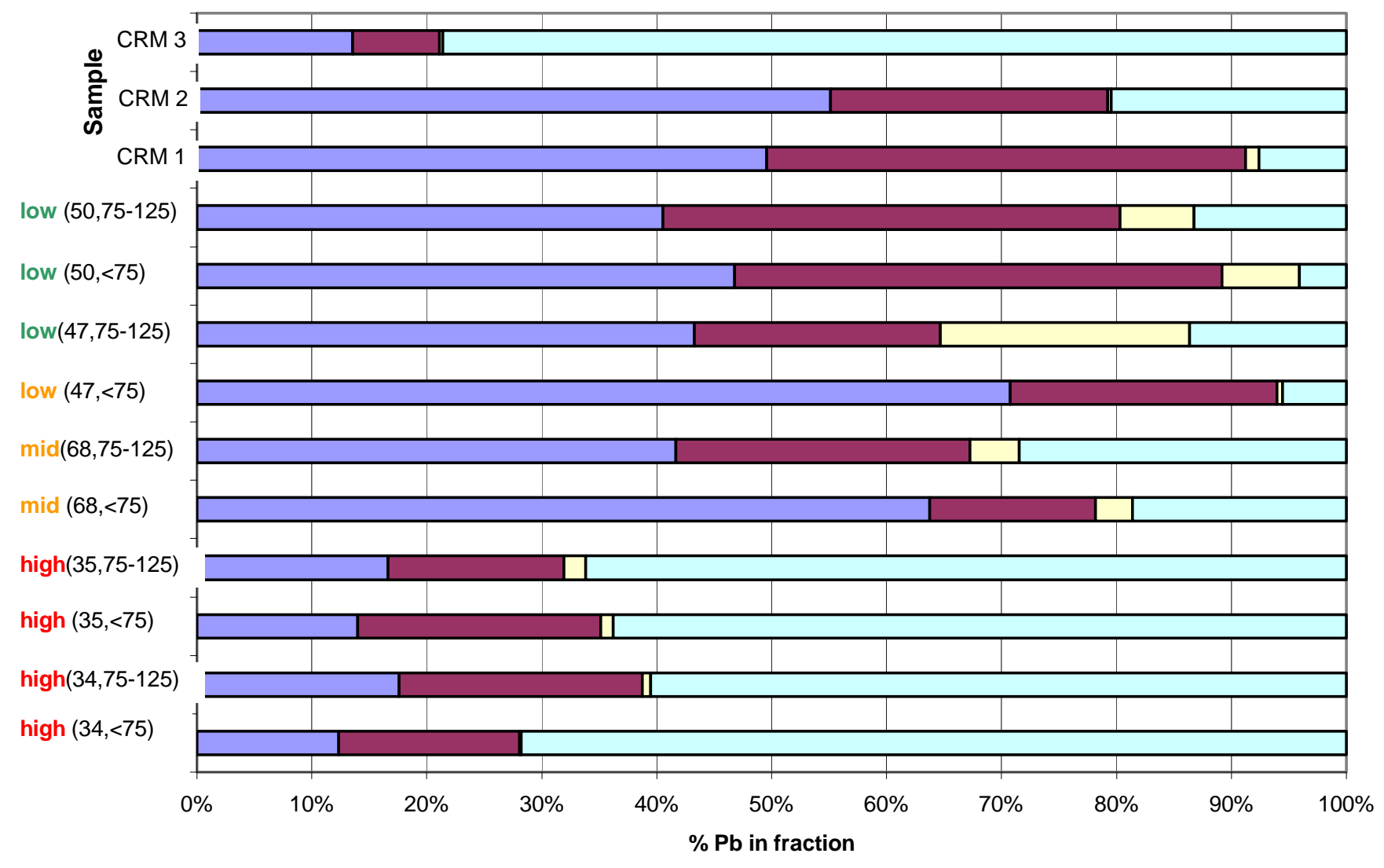

$\square$ Exchangeable $\square F e-M n$ Oxides (clays) $\square$ Organic $\square$ Residual

Fig. 8 


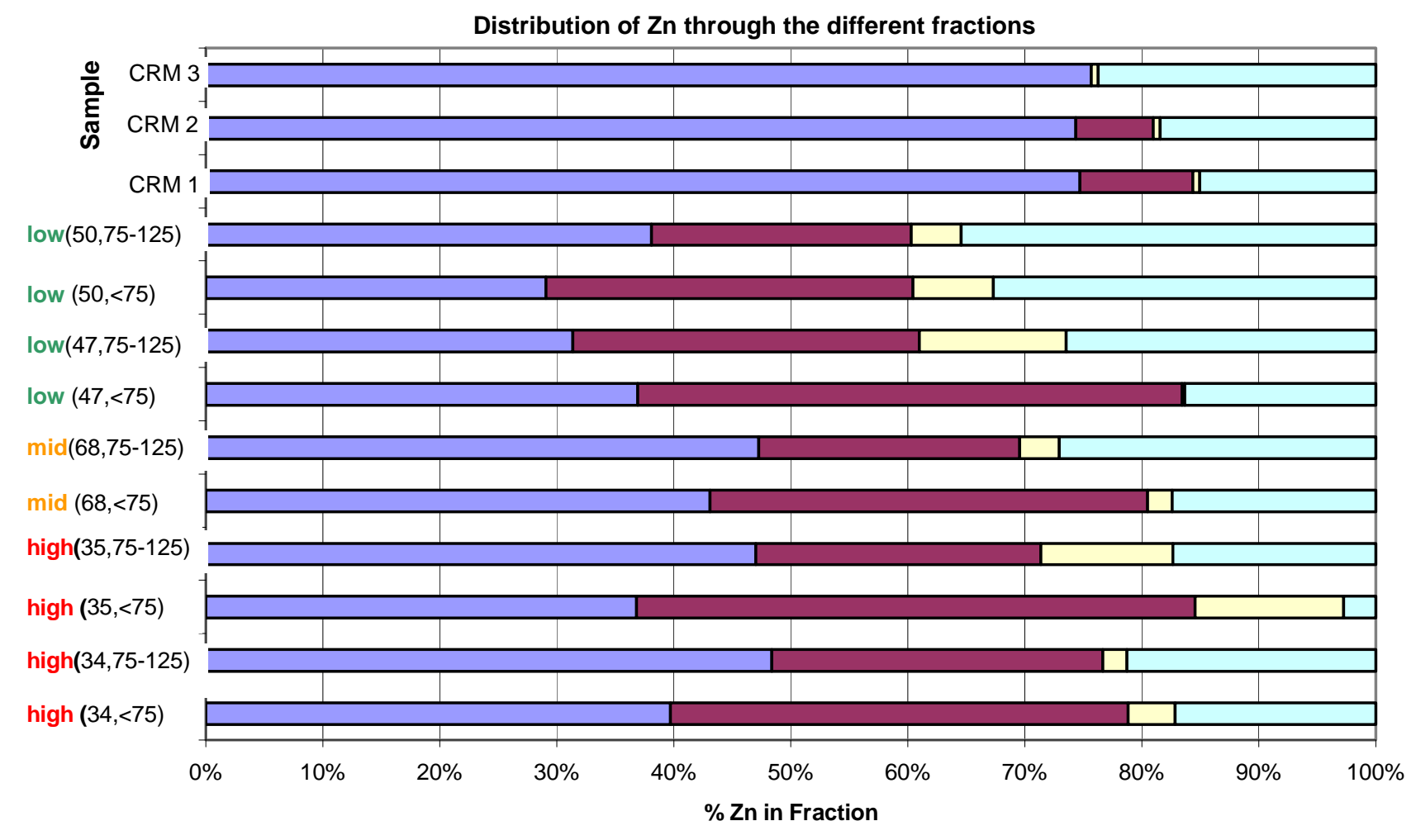

$\square$ Exchangeable $\square \mathrm{Fe}-\mathrm{Mn}$ Oxides (clays) $\square$ Organic $\square$ Residual

Fig. 9 